EPJ Web of Conferences 66, 11012 (2014)

DOI: $10.1051 /$ epjconf/ 20146611012

(C) Owned by the authors, published by EDP Sciences, 2014

\title{
The AGATA commissioning campaign at LNL
}

\author{
D.Mengoni ${ }^{1, a}$ \\ ${ }^{1}$ Dept. of Physics and Astronomy, University of Padova and INFN-Padova
}

\begin{abstract}
The one-year-long commissioning campaign of the AGATA Demonstrator, carried out at the Legnaro National Laboratories, is described, together with the main results achieved. The manuscript is dedicated to the memory of Enrico Farnea.
\end{abstract}

\section{Introduction}

The goal of the AGATA project [1] is the construction of an array of germanium detectors with photopeak efficiency larger than $40 \%$ and peak-to-total ratio larger than $50 \%$. Such values are obtained through the use in real time of pulse shape analysis (PSA) and $\gamma$-ray tracking algorithms. In the initial phase of the project, a subset of the array, composed of five triple clusters, Fig. 1, and known as the AGATA Demonstrator, operated at the Legnaro National Laboratories, with the goal of proving that indeed PSA and $\gamma$-ray tracking can be successfully performed in real time. As described thoroughly in [2], the basic infrastructure of the Demonstrator was ready by the end of 2008. The system was tested and validated with in-beam experiments during 2009.

Commissioning experiments were performed both for testing data acquisition (DAQ) and on-line analysis as well as to quantify and optimize the performance of the array in a stand-alone configuration and in combination with a variety of ancillary detectors, which were used in the subsequent physics campaign.

\section{Commissioning tests}

Preliminary results from the first in-beam test were already reported in [2]. During this test, a beam of ${ }^{30} \mathrm{Si}$ with an energy of $70 \mathrm{MeV}$ was incident on a ${ }^{12} \mathrm{C}$ target, $200 \mu \mathrm{g} / \mathrm{cm}^{2}$ thick. At the time, a single AGATA triple cluster was available, as well as the detector support system, digitizing and pre-processing electronics, global trigger and synchronization system and Narval-based DAQ system. Through a careful examination of the segment spectra, several issues with the cabling and with the detector positioning were solved. The quality of the Doppler correction following PSA and tracking was consistent with a position resolution from the PSA algorithms better than $5 \mathrm{~mm}$ FWHM, formerly established in ref [3].

The second in-beam test was performed in July 2009. In this case, a Coulomb excitation reaction was produced by using ${ }^{56} \mathrm{Fe}$ ions at an energy of $235 \mathrm{MeV}$ on a ${ }^{197} \mathrm{Au}$ target, $1 \mathrm{mg} / \mathrm{cm}^{2}$ thick, which was essentially the same reaction used for the in-beam test of the MARS [4] detector. In this test, one

\footnotetext{
a e-mail: daniele.mengoni@pd.infn.it
} 
available AGATA triple cluster was coupled to an element of DANTE [5], a position-sensitive MCP detector providing the direction of the scattered ions. The AGAVA interface for the auxiliary detectors was not initially available, thus MCP signals were acquired through the available digitizer channels of AGATA. The AGATA trigger processor developed at LNL was first used in-beam, requiring the coincidence between the AGATA detector and the DANTE element. After proper optimization of the signal basis with respect to the crystal orientation axis, a peak FWHM of $3.2 \mathrm{keV}$ was obtained for the $847 \mathrm{keV}$ line of ${ }^{56} \mathrm{Fe}$, which should be compared with the $4.6 \mathrm{keV}$ obtained with the MARS detector and suggests that a position resolution better than $5 \mathrm{~mm}$ FWHM is obtained from the PSA algorithm, namely an adaptive grid search [6].

The third in-beam test was performed in October 2009 with the goals of testing the AGAVA interface on one hand and of evaluating the performance of the AGATA detectors to high-multiplicity events. Two AGATA triple clusters were available, together with detector support system and electronics. For the purpose of testing AGAVA, they were coupled to an array of five 2 in. $x 2$ in. $\mathrm{LaBr}_{3}$ detectors and to a Si-strip detector. This was the first time in which coincidence data could be produced after the tracking process. High-multiplicity data were produced through the fusion-evaporation reaction ${ }^{32} \mathrm{~S}(135 \mathrm{MeV})+{ }^{110} \mathrm{Pd}$, having ${ }^{138} \mathrm{Sm}$ as the main evaporation channel. Through the analysis of data from a 12 hours run with a $1 \mathrm{mg} / \mathrm{cm}^{2}$ thick target with a $12 \mathrm{mg} / \mathrm{cm}^{2}$ gold backing, the transitions from ${ }^{138} \mathrm{Sm}$ were identified up to the $22^{+}$to $20^{+}$transition. This result was compared to the published results by Paul et al. [7], using the EUROGAM-I array and a $300 \mu \mathrm{g} / \mathrm{cm}^{2}$ thick target. The published level scheme extends to higher spins, but it should be noted that in the AGATA data the higher-lying fast transitions will be probably Doppler broadened because of the backed target and they are therefore not visible.

The fourth and fifth in-beam tests were both performed in November 2009 with the goal of debugging the AGATA-PRISMA coupling. In both cases, the ${ }^{58} \mathrm{Ni}(235 \mathrm{MeV})+{ }^{96} \mathrm{Zr}$ multinucleon transfer reaction was performed. The tests were instrumental for solving several bugs with the data acquisition and the data analysis softwar. However, due to the limited statistics acquired, it was not possible to properly evaluate the Doppler correction capabilities of the AGATA-PRISMA setup.

In the following sections, we describe the main achievements of the commissioning experiments are described. A dedicated performance commissioning paper will be forthcoming.

\subsection{AGATA position resolution}

The main goal of the first commissioning run was to test the newly setup DAQ and to measure indirectly the performance of the pulse-shape analysis algorithm under realistic experimental conditions. At this stage of the project, the speed of the PSA algorithm was not considered as an essential parameter for the comparison, and therefore it was decided to store the full information on disk as it is read-out from the preprocessing electronics.

This kind of measurement has already been performed in the past for the MARS [4, 8], GRETA [9] and AGATA [3] detectors. The basic idea is that, when the $\gamma$-rays are emitted in-flight by a recoiling nucleus, the width of peaks in the Doppler-corrected spectra will depend on three factors, namely the intrinsic detector energy resolution, the error on the velocity vector of the emitting nucleus and the uncertainty on the photon direction. The last factor depends on the position resolution of the PSA algorithm used. If the other causes of Doppler broadening are known, the position resolution of the detector can be inferred from the observed energy resolution. This is not an easy task because all the direct and indirect sources of Doppler broadening have to be tracked down and, when not negligible, accurately quantified. 


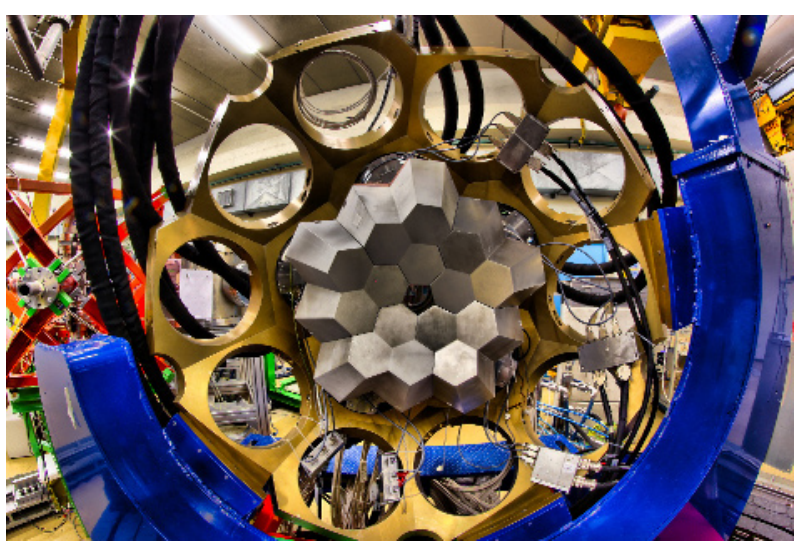

Figure 1. Front view of the AGATA Demonstrator at the Legnaro National Laboratories. The Demonstrator consisted in five triple clusters, more than 500 digital channels, equipped with on-line pulse-shape analysis and tracking.

During this test, a beam of ${ }^{30} \mathrm{Si}$ with an energy of $70 \mathrm{MeV}$ was incident on a ${ }^{12} \mathrm{C}$ target, 200 $\mu \mathrm{g} / \mathrm{cm}^{2}$ thick. The prompt radiation was detected with one AGATA asymmetric triple-cluster detector (ATC1), positioned as close as possible to $90^{\circ}$ with respect to the beam direction.

A full AGATA read-out chain was used to collect data to disk, including AGATA digitizers, Global Trigger and Synchronization, pre-processing electronics and a Narval system [10] running on several nodes. The DAQ chain was started using the Cracow GUI, communicating with the Narval system via a Run Control server. Dedicated Narval actors were performing pulse shape analysis and $\gamma$-ray tracking in real time. As this was the first time in which the full system was running, the original digitized signals were stored to disk for later replay. Because of this, the overall counting rate was limited to approximately $1.5 \mathrm{kHz}$ per crystal, corresponding to approximately $1 \mathrm{~GB}$ of data per minute, per crystal.

Through preliminary data analysis it was possible to spot and to correct for some inconsistencies in the cabling and in the positioning of the detector. The Doppler-corrected spectra for the 1823$\mathrm{keV}$ line of ${ }^{40} \mathrm{~K}$, namely the pn evaporation channel, are shown in Fig. 2. Since no ancillary devices to measure the recoil vector velocity on an event-by-event basis were used, Doppler correction was performed by assuming an average recoil velocity. Using the crystal centre-of-gravity to perform Doppler correction, no peak can be clearly identified corresponding to the $1823-\mathrm{keV}$ transition. This case corresponds to discard the information from the segments, in other words, to treat the detectors as conventional un-segmented coaxial detectors. The peak FWHM is instead $17.7 \mathrm{keV}$ using the segment centre-of-gravity to perform Doppler correction. A peak FWHM of $12.5 \mathrm{keV}$ is obtained when a full PSA algorithm is applied, in this case a grid search algorithm using a signal basis calculated with the JASS code [1]. This ultimate value is consistent with estimates of Doppler broadening using realistic value of the recoil velocity.

The main contribution to the uncertainty on the estimate of the position resolution comes from the difficulty in accounting for all the sources of Doppler broadening in the simulation. Therefore, a different strategy has been followed here in order to extract the position resolution directly from the experimental data in a model-independent way. This was possible because the data were acquired with the detector in two positions, the one having the detector as close as possible to the target, and 

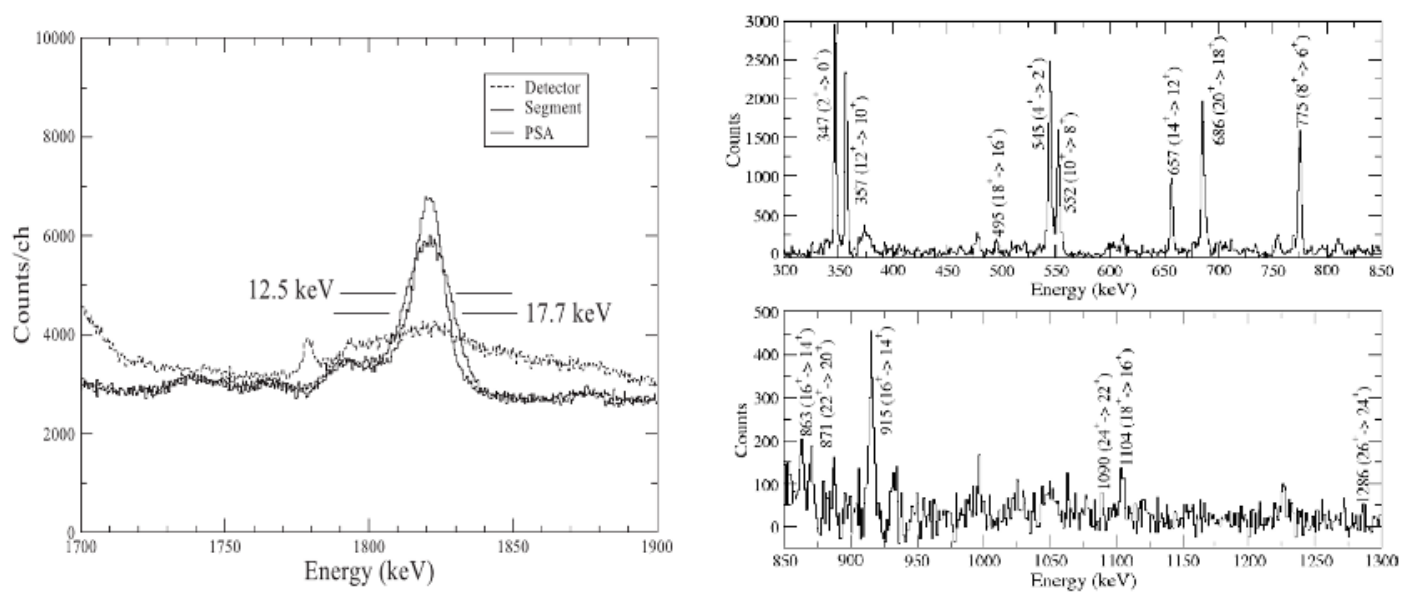

Figure 2. On the left, comparison of Doppler-corrected spectra obtained under different conditions. On the right, example of a sum gate spectra of ${ }^{138} \mathrm{Sm}$. Energy gates were placed on all E2 transitions between $14^{+}$and the ground state $0^{+}$to highlight low-intensity transitions. Although weak, the $1286-\mathrm{keV} 26^{+}$to $24^{+}$can be seen.

the latter having the detector placed farther from the target. Hence, in this experiment, the estimate of the position resolution is reduced to a comparison between the two datasets, since the difference in energy resolution between the Doppler-corrected spectra gathered in the two positions is due to the solid angle subtended by the PSA voxel, i.e. the angular spread on the initial direction of the photon corresponding to the position resolution. From the comparison of the datasets, it is possible to obtain values for the position resolution as a function of the $\gamma$-ray energy. Fig. 2 shows the improvement in the Doppler correction by using different angular resolution, as given by the centre of the detector, the centre of the segment or the PSA result.

The value of the position resolution that was obtained from this analysis suggests that it is of the order of 4.0 - $4.5 \mathrm{~mm}$ for photons with an energy above $1 \mathrm{MeV}$. According to Monte Carlo simulations, this value is adequate to reach the design performance of the AGATA array in terms of efficiency and Doppler correction capabilities.

\subsection{High-multiplicity experiment}

The second test involved two AGATA triple clusters (ATCs), in conjunction with several $\mathrm{LaBr}_{3}(\mathrm{Ce}$ ) and highly segmented silicon strip detector. $\mathrm{Th} \mathrm{LaBr}_{3}$ was supported from the main AGATA, in an arrangement around the two ATCs, whilst the silicon strip detectors were housed inside the reaction chamber at $40 \pm 15$ degrees, with an area of $5 \times 5 \mathrm{~cm}^{2}$. The target rotation was 80 degrees. The ancillary detectors interfaced into the global GTS via AGAVA. The only role of the ancillary detectors in this experiment was to test the AGAVA module. The final AGATA full front-end electronics, preprocessing cards, digitizers and NARVAL based DAQ were in use. On-line analysis was performed after pulse-shape analysis by dedicated NARVAL actors and $\gamma$-ray tracking. The digitized signal traces were also stored for further off-line analysis and processing. The reaction of choice used ${ }^{32} \mathrm{~S}$ beam at $135 \mathrm{MeV}$ incident upon a ${ }^{110} \mathrm{Pd}$ target. The beam intensity was limited to $60 \mathrm{enA}$, and to an overall Ge-detector rate of $8 \mathrm{kHz}$. Data were taken for two different targets: a thin target $\left(500 \mu \mathrm{g} / \mathrm{cm}^{2}\right)$ and a thick target $\left(670 \mu \mathrm{g} / \mathrm{cm}^{2}\right.$ plus $8 \mathrm{mg} / \mathrm{cm}^{2}$ Au backing). Whilst using the thin or thick target different 

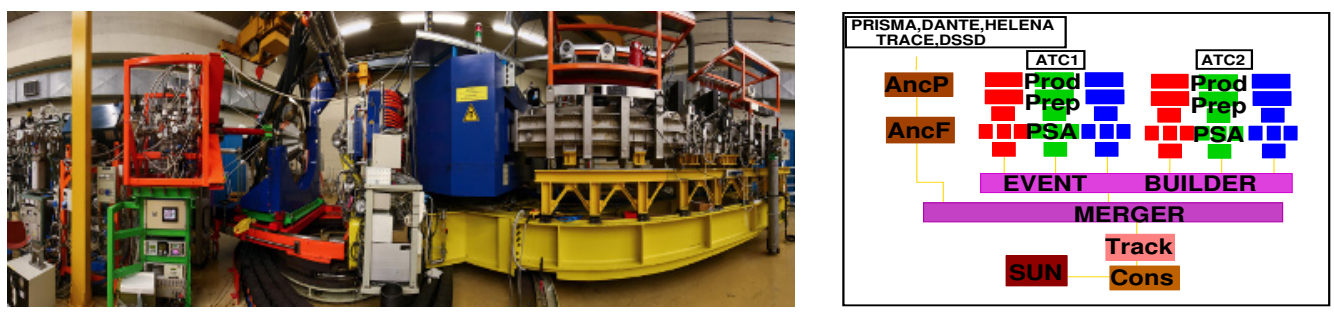

Figure 3. On the left, the AGATA Demonstrator coupled to PRISMA magnetic spectrometer. On the right the data acquisition scheme, which consists in the ancillary and AGATA branches. In the picture it is possible to distinguish, at the local level the data coming from the two triple clusters (ATC1 and ATC2) entering their own processing algorithms (producer, preprocessing and PSA filter) and the ancillary branch with its own producer and preprocessing filter. At the global level an event builder puts together the hits coming from the various detectors, on a timestamped basis, and a merger unit aims at building a global event with correlated gamma-rays and ions.

trigger conditions were employed to record data. In total about $14 \mathrm{~TB}$ of digitized pulse shape data was recorded for off-line analysis.

Analysis of the data was carried out off-line. The digitized signal pulses were replayed through the NARVAL emulator to apply PSA. The MGT tracking algorithm was then used to process the interaction points provided by the PSA algorithm, this applied the Doppler correction and $\gamma$-ray tracking. Only the thin-target data required Doppler corrections, as the emitting nucleus was stopped inside the target in the thick experiment. Therefore no $\gamma$ rays were emitted in flight. $\gamma-\gamma$ matrices were constructed with a modified version of MGT.

In the data, the most intense $\gamma$ rays are associated with the strong output channels of ${ }^{138} \mathrm{Sm}(4 \mathrm{n})$ and ${ }^{138} \mathrm{Pm}(\mathrm{p} 3 \mathrm{n})$, as well as with the Coulomb excitation of the ${ }^{110} \mathrm{Pd}$ target. Previous experiments have studied ${ }^{138} \mathrm{Sm}$ in ${ }^{110} \mathrm{Cd}\left({ }^{32} \mathrm{~S}, 2 \mathrm{p} 2 \mathrm{n}\right){ }^{138} \mathrm{Sm}[11],{ }^{104} \mathrm{Pd}\left({ }^{37} \mathrm{Cl}, \mathrm{p} 2 \mathrm{n}\right){ }^{138} \mathrm{Sm} \mathrm{[12],}{ }^{106} \mathrm{Cd}\left({ }^{35} \mathrm{Cl}, 3 \mathrm{p}\right){ }^{138} \mathrm{Sm} \mathrm{[7]}$ reactions. All of these reactions, being heavy-ion induced, are characterized by the population of highspin states and high multiplicity events. These earlier experiments helped to build the level scheme of ${ }^{138} \mathrm{Sm}$. In the current work, excited states up to spin of $\mathrm{I}^{\pi}=\left(26^{+}\right)$were observed, as shown in the energy spectra of Fig. 2.

Published results in Ref [7], using the EUROGAM-I array and a $300 \mu \mathrm{g} / \mathrm{cm}^{2}$ thick target, extend the level scheme to higher spins, but it should be noted that in the present case the higher-lying fast transitions are probably Doppler broadened because of the backed target and they are therefore not visible.

\subsection{Coupling with complementary detectors}

A series of complementary detectors were used in combination with AGATA, to enhance the available information. The devices underwent a commissioning phase to test their coupling to AGATA with or without the AGAVA VME interface.

\subsubsection{The PRISMA magnetic spectrometer}

PRISMA is a large acceptance magnetic spectrometer designed to work with grazing reactions using heavy-ion beams provided by the LNL accelerators. The basic characteristics of PRISMA are described in Ref. [13] and for the following discussion it is relevant to mention that PRISMA uses ion- 

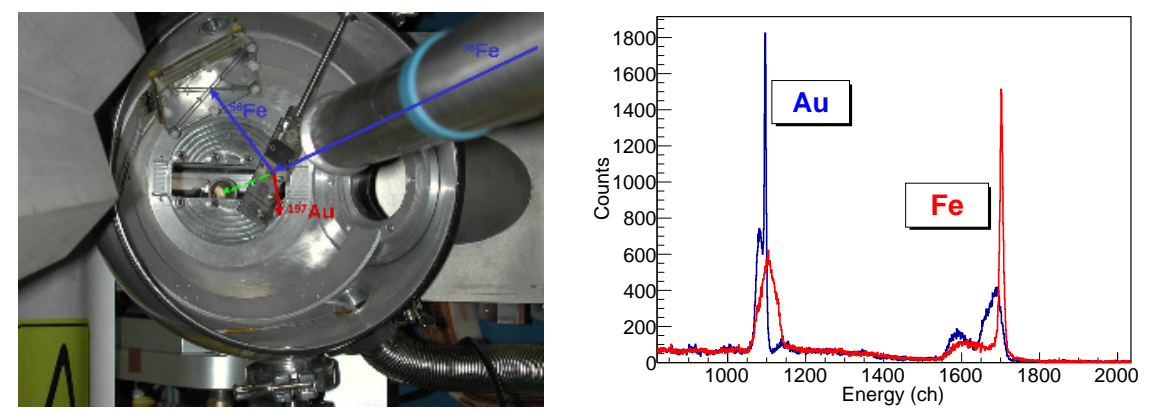

Figure 4. Implementation of the DANTE setup for the commissioning run (left). Doppler corrected peaks, obtained by using the position detected in DANTE and assuming Au or Fe ions alternatively (right).

tracking position-sensitive detectors to achieve good mass resolution. The tracking detectors provide the basic information to obtain the trajectory and velocity of the reaction products [14]. According to Monte Carlo simulations, with velocities up to $\sim 0.1 c$, the intrinsic AGATA detector resolution is almost fully recovered if the recoil velocity is measured with a relative precision better than $1 \%$, and if the recoil velocity direction is measured with a precision better than 1 degree [2]. The AGATA Demonstrator and the PRISMA spectrometer are coupled mechanically onto a platform which can rotate from 0 to 130 degrees, see Fig. 3 . Both devices are fixed on the platform and are located face-to-face in such a way that, independently of the spectrometer angle selected for an experiment, the recoils that enter into the PRISMA spectrometer will have a forward trajectory with respect to the AGATA Demonstrator. The AGATA-PRISMA electronics coupling is obtained via a VME adapter module, AGAVA, which is an interface to the AGATA synchronization unit, the GTS card [15]. Two in-beam commissioning experiments were performed in order to test the coupling of AGATA to the PRISMA magnetic spectrometer via the AGAVA interface. Both of these test experiments used the same beam-and-target combination: a ${ }^{58} \mathrm{Ni}$ beam at $235 \mathrm{MeV}$ incident upon a thin ${ }^{96} \mathrm{Zr}$ target. Following multi-nucleon transfer reactions at the target position, the beam like products were transported to the focal plane of PRISMA where they were identified by $\mathrm{Z}$ and $\mathrm{A}$. The first of these tests took place in November 2009. The set-up consisted in two triple clusters plus a DANTE MCP and the PRISMA magnetic spectrometer both acquired via standard VME electronics and coupled through the AGAVA interface. The second in-beam test with the PRISMA spectrometer was carried out in December 2009. The reaction and experimental set-up used, was the same as for the previous experiment. The purpose of the second experiment was the optimization of the on-line PRISMA analysis, and to collect more data to investigate the performance of the AGATA-PRISMA coupling.

One of the major improvements in the second of these experiment was that it was possible to carry out the full PRISMA reconstruction on-line. It was possible to process the PRISMA information and track the ions entering the spectrometer via a dedicated library, loaded into the ancillary filter actor of the DAQ system, Fig. 3 (right). The recoil velocity, obtained after the full trajectory reconstruction, was used for a real-time Doppler correction of the gamma-tracked spectrum. However, the lack of statistics didn't allow for a proper estimation of the best achievable Doppler correction, reachable using AGATA in combination with PRISMA. 

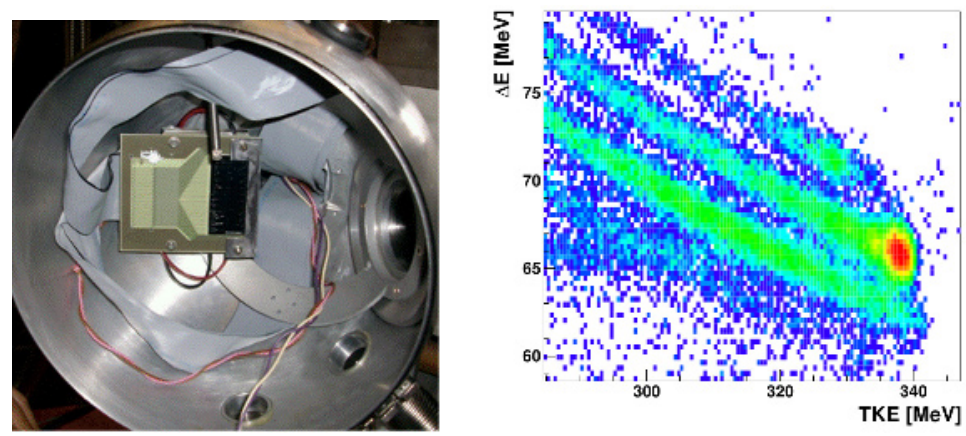

Figure 5. Left: TRACE first commissioning setup. Right: E- $\Delta \mathrm{E}$ matrix obtained from the energy detected in the TRACE telescopes. The mass separation of the oxigen ions $(A=16,17$ and 18$)$ detected by the silicon telescope is evident.

\subsubsection{The DANTE micro-channel plate}

All the AGATA events correlated with reaction products, which are outside the PRISMA acceptance, are lost during the analysis. In order to recover a fraction of those events, the DANTE [5, 16] positionsensitive array is also coupled to the AGATA-PRISMA setup. This detector performs the Doppler correction of the prompt $\gamma$ rays detected in AGATA event by event, with an average ejectile velocity estimated from a two-body kinematics.

DANTE is a heavy-ion position-sensitive ancillary array based on Micro-Channel Plates (MCP), installed in the reaction chamber of the AGATA-PRISMA setup. The micro-channel plates present a configuration very similar to that of the start detector [17] of the PRISMA spectrometer. Each detector consists of a mylar foil, at the entrance, for electron production, followed by two MicroChannel-Plates (MCP), of dimensions $40 \times 60 \mathrm{~mm}^{2}$, mounted in Chevron configuration.

A Coulomb excitation reaction was induced using ${ }^{56} \mathrm{Fe}$ ions at an energy of $235 \mathrm{MeV}$ incident on a ${ }^{197} \mathrm{Au}$ target, $1 \mathrm{mg} / \mathrm{cm}^{2}$ thick. One AGATA triple cluster was coupled to an element of DANTE. Since the AGAVA interface for the auxiliary detectors was not yet available, the MCP signals were acquired through the available digitizer channels of AGATA. The AGATA trigger processor developed at LNL was first used in-beam, requiring the coincidence between the AGATA detector and the DANTE element. After proper optimization of the signal basis with respect to the crystal orientation axis, a peak FWHM of $3.2 \mathrm{keV}$ was obtained for the $847-\mathrm{keV}$ line of ${ }^{56} \mathrm{Fe}$, see Fig. 4 (right), which should be compared with the $4.6 \mathrm{keV}$ obtained with the MARS detector and suggests that indeed a position resolution better than $5 \mathrm{~mm}$ FWHM is obtained from the PSA algorithm, namely an adaptive grid search.

\subsubsection{The TRACE highly-segmented silicon array}

In preparation for experiments with the AGATA Demonstrator array, see contribution of F.Crespi et al. in this conference proceedings, a test run was performed in June 2009 to verify the performance of a TRACE Si telescope [2] that was afterwards employed during the physics campaign, to measure with high resolution ${ }^{17} \mathrm{O}$ scattered ions to enhance, by gating on the total kinetic energy loss, the population of resonant states.

TRACE is a project which aims at the development of highly-segmented silicon array for direct and fusion-evaporation reaction, which provides a high detection efficiency for the use of the digital 

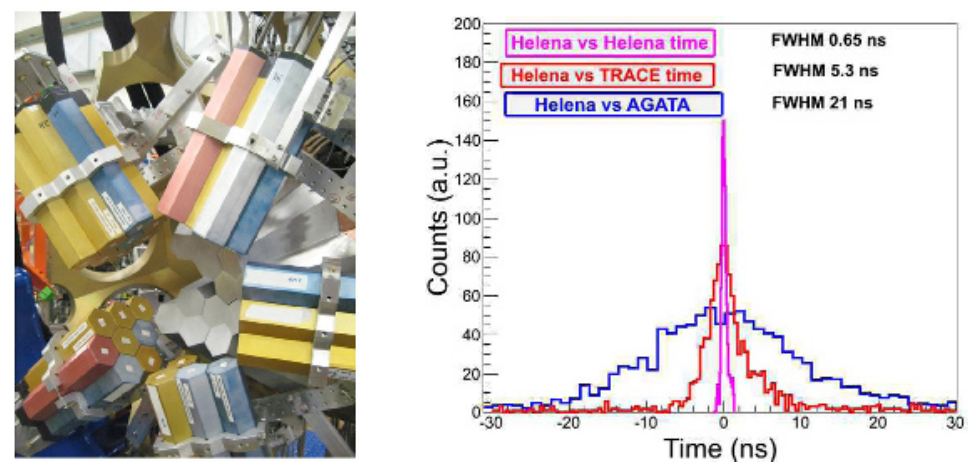

Figure 6. On the left, the experimental setup used in the ${ }^{174} \mathrm{~W}$ experiment [22], consisting of four triple clusters of AGATA (in the back of the picture) and an array of $27 \mathrm{Ba} F_{2}$ detectors, grouped in five clusters $(8+8+4+4+3$ detectors). On the right, the time spectra obtained by using HELENA crystals in coincidence with other detectors: HELENA, TRACE and AGATA. The quality of the intrinsic time resolution, $650 \mathrm{ps}$, is evident in comparison with the timing properties of the semiconductor detectors.

pulse-shape analysis technique. The advantages of pulse shape analysis (PSA) in comparison to time of flight, $\Delta \mathrm{E}-\mathrm{E}$ techniques, or other methods [18] are well established. Several detector systems are being built in Europe such as, in addition to TRACE, FAZIA, GASPARD and HYDE [19-21] to achieve an efficient and low-cost particle-identification system.

The aim of the experiment was to measure with high resolution the $\gamma$-decay of giant resonances which were excited by inelastic scattering of ${ }^{17} \mathrm{O}$ ions, at $20 \mathrm{MeV} / \mathrm{A}$. Previously, low-resolution experiments have shown that the decay of giant resonances is largely dominated by the isovector giant dipole resonance (GDR) at high bombarding energies, whereas the quadrupole resonance is the stronger excitation mode at lower bombarding energies (around $20 \mathrm{MeV} / \mathrm{A}$ ).

In the AGATA Demonstrator test, the scattered ${ }^{17} \mathrm{O}$ ions were detected by two segmented silicon $\Delta \mathrm{E}-\mathrm{E}$ telescopes, cooled to -20 Celsius degrees, made of detectors $200 \mu \mathrm{m}$ and $1000 \mu \mathrm{m}$ hick. Each detector had an active area of $20 \times 50 \mathrm{~mm}^{2}$ divided in 60 pads of $4 \times 4 \mathrm{~mm}^{2}$, half of which were connected to the electronics and read out. The telescopes, mounted symmetrically with respect to the beam axis, were placed at 12 degrees for the ${ }^{208} \mathrm{~Pb}$ reaction and at 8 degrees for the ${ }^{90} \mathrm{Zr}$ reaction. The isotopic separation are clearly visible in Fig. 5.

\subsubsection{The HELENA multiplicity filter}

In the experiment described in the previous section, several $\mathrm{BaF}_{2}$ modules for high-energy $\gamma$ detection were also in place, as part of the HELENA array [2].

The HELENA array is used both as a time reference and a multiplicity filter for $\gamma$-rays. HELENA is a detector array built out of 27 scintillation detectors based on 3 in. $x 3$ in. hexagonal $\mathrm{BaF}_{2}$ crystals. These detectors are placed at $150 \mathrm{~mm}$ from the target, in five groups of eight, eight, four, four and three detectors each, to cover the maximum available space between AGATA and the PRISMA spectrometer. The solid-angle coverage is approximately $25 \%$ of total solid angle with an overall full-energy peak efficiency of $16 \%$ at $500 \mathrm{keV}$. 
The complete setup, implemented during the physics campaign and succesfully used in ${ }^{174} \mathrm{~W}$ experiment reported in ref [22], is presented in Fig. 6, alongside with the excellent timing properties of the scintillator, in self-coincidence or in coincidence either with AGATA or TRACE.

\section{Summary and conclusions}

In this work we reviewed the commissioning campaign run of the first implementation of the AGATA spectrometer, consisting of 5 triple clusters and installed at the Legnaro National Laboratories. The main results, among with the position resolution, high-spin performance, and coupling with complementary detectors (PRISMA, DANTE, TRACE, HELENA) are presented and discussed.

The commissioning campaign was essential to prepare the system for the subsequent two-yearlong physics campaign.

\section{Acknowledgments}

The author is grateful to the AGATA and PRISMA Collaborations. This work has been supported by the Istituto Nazionale di Fisica Nucleare (Italy), by the Science and Technology Facilities Council (UK) and by BMBF, Germany, under Grants 06K-167 and 06KY205I.

\section{References}

[1] S. Akkoyun et al., Nucl. Ins. Meth. A668, 26-58 (2012).

[2] A. Gadea et al., Nucl. Ins. Meth. A654, 88-96 (2011).

[3] F. Recchia et al., Nucl. Ins. Meth. A604, 555-562 (2009).

[4] T. Kroell et al., Nucl. Ins. Meth. A565, 691-703 (2006).

[5] J.J. Valiente-Dobón et al., Fusion06, AIP conference proceedings, 202, 2006.

[6] P.-A. Söderström et al., Nucl. Ins. Meth. A638, 96-109 (2011).

[7] E. Paul et al., J. Phys G: Nucl. Part. Phys. 20, 1405 (1994).

[8] T. Kroell et al., Nucl. Ins. Meth. A586, 421-431 (2008).

[9] M. Descovich et al., Nucl. Ins. Meth. A553, 535-542 (2005).

[10] X. Grave et al, 14th IEEE-NPSS, 1 (2005).

[11] S. Lunardi et al., Z. Phys. A321, 177 (1985).

[12] E. Paul et al., Phys. Rev. C36, 2380 (1987).

[13] A.M. Stefanini et al., Nucl.Phys. A701, 217c (2002).

[14] D. Montanari et al., Eur. Phys. J. A47, 4 (2011).

[15] M. Bellato et al., JINST 8, P07003 (2013).

[16] A. Gottardo et al. Nuclear Physics A805, 606 (2008).

[17] G. Montagnoli et al., Nucl. Ins. Meth. A547, 455 (2005).

[18] L. Bardelli, et al., Nuclear Physics A746, 272 (2004).

[19] J. Dueñas et al., Nucl. Ins. Meth. A676, 70-73 (2012).

[20] J. Dueñas et al., Nucl. Ins. Meth. A714, 48-52 (2013).

[21] B. Genolini et al., in press, http://dx.doi.org/10.1016/j.nima.2013.06.078

[22] V.Vandone et al, Phys. Rev. C88, (2013) 034312. 
